\title{
A derived neutrophil to lymphocyte ratio predicts survival in patients with cancer
}

\author{
MJ Proctor ${ }^{*, 1}$, DC McMillan', DS Morrison ${ }^{2}$, CD Fletcher ${ }^{3}$, PG Horgan' and SJ Clarke \\ 'University Department of Surgery, Faculty of Medicine, Royal Infirmary, University of Glasgow, Glasgow G3I 2 ER, UK; ${ }^{2}$ Faculty of Medicine, West of \\ Scotland Cancer Surveillance Unit, University of Glasgow, Glasgow GI 2 8RZ, UK; ${ }^{3}$ Department of Clinical Biochemistry, Royal Infirmary, Glasgow G4 OSF, UK; \\ ${ }^{4}$ Department of Medical Oncology, Royal North Shore Hospital, St Leonards, 2065, New South Wales, Australia
}

BACKGROUND: The neutrophil lymphocyte ratio (NLR) has prognostic value in patients with a variety of cancers. Many chemotherapeutic trial databases hold information on white cell and neutrophil counts only. The aim of the present study was to compare the prognostic value of the NLR with a derived score (dNLR), composed of white cell and neutrophil counts.

METHODS: Patients $(n=27031)$ who were sampled incidentally between 2000 and 2007 for neutrophil, lymphocyte and white cell counts, and also had a diagnosis of cancer (Scottish Cancer Registry), were identified. Of this group, I2 I I 8 patients who had been sampled within 2 years of their cancer diagnosis were studied.

RESULTS: On follow-up, there were 7366 deaths, of which 6198 (84\%) were cancer deaths. The median time from blood sampling to diagnosis was 2.1 months. The area under the receiver-operating characteristic (ROC) curve for cancer-specific survival was 0.650 for the NLR and 0.640 for the dNLR. The NLR and dNLR were independently associated with survival in all cancers studied (all $P<0.00$ I). The optimal thresholds, on the basis of hazard ratios and area under the curve, were $4:$ I for the NLR and $2:$ I for the dNLR.

CONCLUSION: The results of the present study show that the $d N L R$ has similar prognostic value to the NLR. Therefore, the universally available dNLR is to be commended for use in the risk stratification of patients undergoing chemotherapy.

British Journal of Cancer (2012) 1 07, 695-699. doi:I0.1038/bjc.2012.292 www.bjcancer.com

Published online 24 July 2012

(c) 2012 Cancer Research UK

Keywords: neutrophil; lymphocyte; prognosis; survival analysis; oncology

Cancer incidence is increasing in the United Kingdom, the United States and worldwide (Ferlay, 2010). Although it is clear that the development of cancer has a genetic basis, recent work has demonstrated that the host inflammatory response plays an important role in carcinogenesis and disease progression (Colotta et al, 2009; Hanahan and Weinberg, 2011).

It is therefore of interest that the combination of haematological components of the systemic inflammatory response, specifically neutrophils and lymphocytes, termed the neutrophil lymphocyte ratio (NLR) have been shown to have prognostic value in patients with a variety of cancers (Walsh et al, 2005; Cho et al, 2009; Sarraf et al, 2009; Shimada et al, 2010; Azab et al, 2011; Sharaiha et al, 2011), as well as patients undergoing chemotherapy for cancer (Kao et al, 2010; Chua et al, 2011). Although, apparently inferior to other measures of the systemic inflammatory response, such as the mGPS (Proctor et al, 2011a), the NLR does have the advantage of its components being inexpensive and routinely measured in dayto-day oncological practice, and in current chemotheraputic cancer trials. Clearly, if such extensive data were to confirm the prognostic value and clinical utility of the NLR, this would be an important, relevant, clinical translational advance in the identification of cancer patients at high risk (Clarke et al, 2011).

*Correspondence: MJ Proctor; E-mail: michael.j.proctor@gmail.com Received 5 March 2012; revised 6 June 2012; accepted 12 June 2012; published online 24 July 2012
However, on patient entry to chemotherapeutic trials, despite having a differential white cell count carried out, only white cell and neutrophil counts are routinely entered into clinical trial databases. In an attempt to obviate this problem and allow the widespread utilisation of a similar inflammation-based score in such settings, we aimed to investigate the prognostic value of a derived NLR (dNLR), from a white cell and neutrophil count. Therefore, the aim of the present study was to compare the prognostic value of the NLR and dNLR adjusted for age, sex, deprivation and tumour site in the Glasgow Inflammation Outcome Study (GIOS).

\section{PATIENTS AND METHODS}

\section{Study design}

From the GIOS cohort previously described (Proctor et al, 2010), patients who had samples including a differential white cell count (white cell count, neutrophil count and lymphocyte count) were included. At the time of data collection, the Scottish Cancer Registry (SCR) held complete pathological and clinical cancer diagnosis records from 1 January 1980 until 31 December 2007, and mortality follow-up until 30 June 2009. Deaths were classed as cancer-specific if the primary cause of death matched the primary cancer diagnosis. Otherwise, deaths were classed as non-cancerspecific. Cancer stage data was obtained from the SCR where available. 
Table I The relationship between patient characteristics, tumour site, and overall and cancer-specific survival in all patients

\begin{tabular}{|c|c|c|c|c|c|c|}
\hline & & $n=12|| 8(\%)$ & $\begin{array}{l}\text { 5-year overall survival \% } \\
\text { ( } n \text { of deaths) } n=7366\end{array}$ & $P$-value & $\begin{array}{c}\text { 5-year cancer specific survival \% } \\
\text { ( } n \text { of deaths) } n=6198\end{array}$ & $P$-value \\
\hline \multirow[t]{2}{*}{ Age } & $<65$ years & $5423(45)$ & $50(257 \mid)$ & & $54(23 \mid 4)$ & \\
\hline & $\geqslant 75$ years & $3022(25)$ & $20(2383)$ & $<0.001$ & $30(1843)$ & $<0.001$ \\
\hline \multirow[t]{2}{*}{ Sex } & Male & 5900 (49) & $28(4094)$ & & $35(3435)$ & \\
\hline & Female & $6218(5 \mathrm{I})$ & $46(3272)$ & $<0.001$ & $52(2763)$ & $<0.001$ \\
\hline & 2 & $1485(12)$ & $45(780)$ & & $51(654)$ & \\
\hline & 3 & $1804(15)$ & $42(1011)$ & & $48(866)$ & \\
\hline & 4 & $2439(20)$ & $34(154 \mid)$ & & $42(1284)$ & \\
\hline & 5 (most deprived) & $4693(39)$ & $30(3175)$ & $<0.001$ & $38(2663)$ & $<0.001$ \\
\hline \multirow[t]{6}{*}{ Tumour site } & Breast & $2147(18)$ & 78 (442) & & $84(304)$ & \\
\hline & Bladder & $562(5)$ & $48(286)$ & & $62(195)$ & \\
\hline & Colorectal & $1413(12)$ & $39(820)$ & & $46(680)$ & \\
\hline & Head and Neck & $738(6)$ & $35(452)$ & & $52(296)$ & \\
\hline & Hepatopancreaticobiliary & $721(6)$ & $6(660)$ & & $7(626)$ & \\
\hline & Pulmonary & $2343(19)$ & $6(2177)$ & $<0.001$ & 8 (2004) & $<0.001$ \\
\hline
\end{tabular}

Abbreviation: SIMD = Scottish Index of Multiple Deprivation.

Patients with blood samples taken within 2 years of their cancer diagnosis were included in the analysis, and split into those sampled before and following cancer diagnosis. The dNLR was derived from the assumption that the white cell count is made up primarily of lymphocytes and neutrophils, and therefore, the white cell count minus the neutrophil count would be broadly similar to the lymphocyte count. As different thresholds have been suggested in the past (Ding et al, 2010; Kim et al, 2010; Ohno et al, 2010; Sharaiha et al, 2011), several were examined to ascertain the optimal NLR and dNLR.

Patient inclusion criteria has been previously detailed and only cancer groups previously studied were included (Proctor et al, 2010). Ethical approval was granted for the present study by the Research Ethics Committee, North Glasgow NHS Trust.

\section{Methods}

The North Glasgow haematological database was searched to obtain patients with white cell, neutrophil and lymphocyte counts. The NLR was constructed as follows: NLR = neutrophil count to lymphocyte count. The dNLR was constructed as follows: $\mathrm{dNLR}=$ neutrophil count to (white cell count - neutrophil count).

International Classification of Disease 10 codes were used to identify the site of cancer diagnosis as previously described (Proctor et al, 2010). The Scottish Index of Multiple Deprivation (SIMD) 2006, as recommended by the Information Services Division on behalf of NHS Scotland and the Scottish Government Department of Health, was used to measure deprivation with the least deprived being scored as 1 to the most deprived scoring 5 (Bishop et al, 2004).

\section{Statistics}

Survival, overall and cancer-specific, was calculated from the time of cancer diagnosis to death. Kaplan-Meier estimator was used to analyse the relationship between patient characteristics, tumour site, and overall and cancer-specific survival (Table 1).

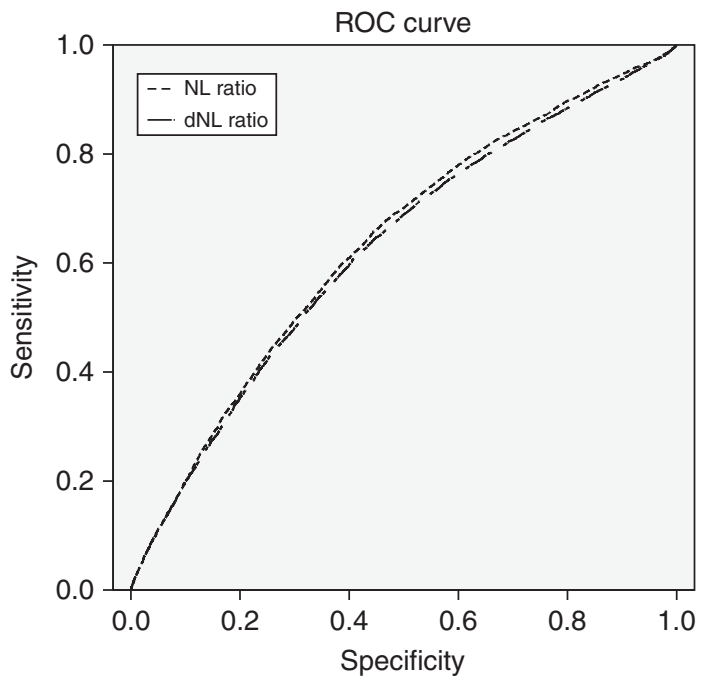

Figure I Receiver-operating characteristic curve for cancer-specific survival.

Receiver-operating characteristic (ROC) curve was used to determine the sensitivity and specificity similarities between the NLR and dNLR (Figure 1). Cox proportional multivariate regression analysis, corrected for age, sex, deprivation and tumour site, as well as area under the ROC curve, were used to determine the relationship between different NLR and dNLR thresholds (in whole numbers) and survival in patients sampled before and after diagnosis (Table 2). Box plot was used to demonstrate the relationship between the NLR, dNLR and Dukes stage in patients with colorectal cancer (Figure 2). Cox proportional multivariate regression analysis, corrected for age, sex, deprivation and tumour site, as well as area under ROC curve, were used to determine the relationship between optimal NLR and dNLR thresholds, 
Table 2 The relationship between NLR and dNLR thresholds, and survival. Adjusted for age, sex, deprivation and stratified by tumour site

\begin{tabular}{|c|c|c|c|c|c|c|c|c|}
\hline & \multirow[b]{2}{*}{ Ratio } & \multirow[b]{2}{*}{ n (\%) } & \multicolumn{3}{|c|}{ Overall survival } & \multicolumn{3}{|c|}{ Cancer-specific survival } \\
\hline & & & HR & $P$-value & AUC & HR & $P$-value & AUC \\
\hline \multicolumn{9}{|c|}{ Patient sampled prior to cancer diagnosis, $n=3859$} \\
\hline \multirow[t]{10}{*}{ NLR } & $<1: 1$ & $78(2)$ & 1 & & & 1 & & \\
\hline & $1: 1$ & 3781 (98) & 1.03 & 0.840 & 0.507 & 0.967 & 0.859 & 0.507 \\
\hline & $<2: 1$ & $589(15)$ & I & & & 1 & & \\
\hline & $\geqslant 2: 1$ & $3270(85)$ & 1.49 & $<0.001$ & 0.563 & 1.48 & $<0.001$ & 0.547 \\
\hline & $<3: 1$ & $1354(35)$ & I & & & 1 & & \\
\hline & $\geqslant 3: 1$ & $2505(65)$ & 1.55 & $<0.001$ & 0.593 & 1.52 & $<0.001$ & 0.560 \\
\hline & $<4: 1$ & $1987(51)$ & 1 & & & 1 & & \\
\hline & $\geqslant 4: 1$ & $1872(49)$ & 1.57 & $<0.001$ & 0.598 & 1.52 & $<0.001$ & 0.558 \\
\hline & $<5: 1$ & $2435(63)$ & I & & & 1 & & \\
\hline & & 1424 (37) & 1.50 & $<0.001$ & 0.579 & 1.44 & $<0.001$ & 0.542 \\
\hline \multirow[t]{10}{*}{$d N L R$} & $<1: 1$ & $214(6)$ & 1 & & & 1 & & \\
\hline & $\geqslant 1: 1$ & $3645(94)$ & 1.17 & 0.098 & 0.518 & 1.16 & 0.163 & 0.516 \\
\hline & $<2: 1$ & $1399(36)$ & I & & & 1 & & \\
\hline & $\geqslant 2: 1$ & $2460(64)$ & 1.54 & $<0.001$ & 0.593 & 1.53 & $<0.001$ & 0.563 \\
\hline & & $2461(6$ & I & & & & & \\
\hline & $\geqslant 3: 1$ & $1398(36)$ & 1.47 & $<0.001$ & 0.575 & 1.43 & $<0.001$ & 0.543 \\
\hline & $<4: 1$ & $2960(77)$ & 1 & & & 1 & & \\
\hline & $\geqslant 4: 1$ & $899(23)$ & 1.46 & $<0.001$ & 0.552 & 1.41 & $<0.001$ & 0.527 \\
\hline & $<5: 1$ & $3267(85)$ & । & & & 1 & & \\
\hline & $\geqslant 5: 1$ & $592(15)$ & 1.40 & $<0.001$ & 0.538 & 1.33 & $<0.001$ & 0.517 \\
\hline \multicolumn{9}{|c|}{ Patient sampled following cancer diagnosis, $n=8259$} \\
\hline \multirow[t]{10}{*}{ NLR } & $<1: 1$ & 296 (4) & I & & & 1 & & \\
\hline & $\geqslant 1: 1$ & $7963(96)$ & 1.16 & 0.094 & 0.505 & 1.89 & 0.069 & 0.506 \\
\hline & $<2: 1$ & | 480 (I8) & I & & & 1 & & \\
\hline & $\geqslant 2: 1$ & $6779(82)$ & 1.63 & $<0.001$ & 0.575 & 1.67 & $<0.001$ & 0.566 \\
\hline & $<3: 1$ & $2984(36)$ & I & & & 1 & & \\
\hline & $\geqslant 3: 1$ & $5275(64)$ & 1.85 & $<0.001$ & 0.641 & 1.93 & $<0.001$ & 0.629 \\
\hline & $<4: 1$ & $4104(50)$ & I & & & 1 & & \\
\hline & $\geqslant 4: 1$ & 415 & 1.86 & $<0.001$ & 0.661 & 1.92 & $<0.001$ & 0.646 \\
\hline & $<5: 1$ & $4872(59)$ & I & & & I & & \\
\hline & $\geqslant 5: 1$ & $3387(41)$ & 1.82 & $<0.001$ & 0.657 & 1.86 & $<0.001$ & 0.642 \\
\hline \multirow[t]{10}{*}{$d N L R$} & $<1: 1$ & $655(8)$ & 1 & & & I & & \\
\hline & $\geqslant 1: 1$ & 7604 (92) & 1.35 & $<0.001$ & 0.521 & 1.39 & $<0.001$ & 0.519 \\
\hline & $<2: 1$ & 3083 (37) & I & & & 1 & & \\
\hline & $\geqslant 2: 1$ & $5176(63)$ & 1.76 & $<0.001$ & 0.630 & 1.8 & & 0.620 \\
\hline & $<3: 1$ & $4978(60)$ & I & & & I & & \\
\hline & $\geqslant 3: 1$ & $3281(40)$ & 1.74 & $<0.001$ & 0.644 & 1.78 & $<0.001$ & 0.631 \\
\hline & $<4: 1$ & 6034 (73) & 1 & & & I & & \\
\hline & $\geqslant 4: 1$ & $2225(27)$ & 1.75 & $<0.001$ & 0.619 & 1.78 & $<0.001$ & 0.609 \\
\hline & $<5: 1$ & $6632(80)$ & I & & & I & & \\
\hline & $\geqslant 5: 1$ & $1627(20)$ & 1.76 & $<0.001$ & 0.595 & 1.80 & $<0.001$ & 0.589 \\
\hline
\end{tabular}

Abbreviations: $A \cup C=$ area under receiver-operating characteristic curve; $d N L R=$ derived NLR; HR = hazards ratio; NLR = neutrophil lymphocyte ratio. Shaded values signify the optimal thresholds. and survival in patients with advanced (Dukes C and D) colorectal cancer (Table 3). Analysis was performed using SPSS software (SPSS Inc., Chicago, IL, USA).

\section{RESULTS}

From the GIOS cohort of 223303 patients previously described (Proctor et al, 2010), 27031 patients were identified as also having a diagnosis of cancer. Within this group, 12118 patients had been sampled within 2 years of their cancer diagnosis. Of this group, $3859(32 \%)$ were sampled before a diagnosis and 8259 (68\%) were sampled following diagnosis, and the possible initiation of surgical or chemotherapeutic interventions.

The majority of patients were under the age of 75 years $(n=9096,75 \%)$, were female $(n=6218,51 \%)$ and were from the most deprived SIMD quintile $(n=4693,39 \%)$. The minimum follow-up from cancer diagnosis was 18 months and the maximum 132 months (median 52 months for survivors).

The relationship between patient characteristics, tumour site, and overall and cancer-specific survival in all patients is shown in Table 1. In total, 12118 patients were studied. On follow-up, there were 7366 deaths of which $6198(84 \%)$ were cancer-related. The median time from blood sampling to diagnosis was 1.6 months in those sampled before diagnosis and 2.2 months in those sampled following diagnosis, suggesting that most scores reflect status at diagnosis. Increasing age, male gender and increasing deprivation were associated with reduced 5-year overall and cancer-specific survival (all $P<0.001$ ).

The ROC curves, using cancer-specific death as an end-point $(n=12118)$ for NLR and dNLR is shown in Figure 1. The ROC curves for NLR and dNLR were $0.650(P<0.001)$ and 0.640 $(P<0.001)$, respectively. The Spearman rank correlation between the NLR and dNLR was $0.962(P<0.001)$.

The relationship between NLR and dNLR thresholds and survival in patients sampled before and following diagnosis, adjusted for age, sex and deprivation, and stratified by tumour site, is shown in Table 2. The optimal thresholds for the NLR $(\geqslant 4: 1)$ and the dNLR $(\geqslant 2: 1)$ in both patients sampled before and following diagnosis, as measured by the hazard ratios and area under the ROC curve, are highlighted.

In the present cohort, a limited number of patients had stagerelated data available in the SCR, with only colorectal cancer having stage data for over $50 \%$ of patients. Stage was only available in $29 \%(n=621)$ of patients with breast cancer and $10 \%(n=234)$ of patients with pulmonary cancer. Other cancer groups had no stage available. Of the colorectal cancer patients with stage available, 90 (9\%) were Dukes A, $264(27 \%)$ were Dukes B, 327 (33\%) were Dukes C and $312(31 \%)$ were Dukes D.
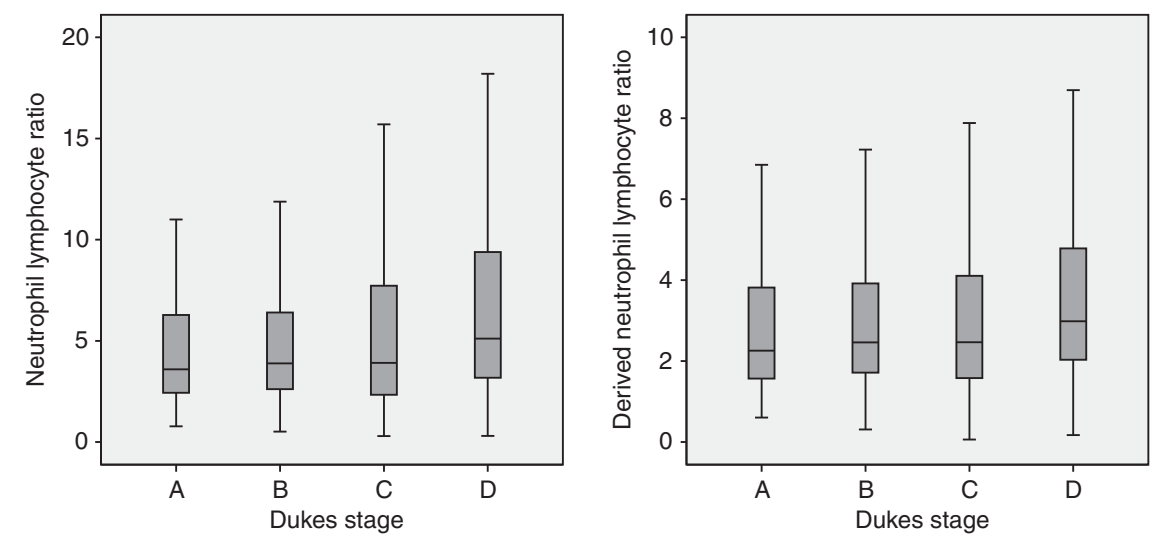

Figure 2 The relationship between NLR, dNLR and Dukes stage in patients with colorectal cancer. 
Table 3 The relationship between the NLR, dNLR and survival in patients with advanced colorectal cancer (Dukes $C$ and D). Adjusted for age, sex and deprivation

\begin{tabular}{|c|c|c|c|c|c|c|c|c|}
\hline & \multicolumn{4}{|c|}{ Overall survival } & \multicolumn{3}{|c|}{$\begin{array}{l}\text { Cancer-specific } \\
\text { surviva }\end{array}$} \\
\hline & & $n=639(\%)$ & HR & $P$-value & AUC & HR & $P$-value & AUC \\
\hline \multirow[t]{2}{*}{ NLR } & $<4: 1$ & $278(44)$ & 1 & & & 1 & & \\
\hline & $\geqslant 4: 1$ & $361(56)$ & 1.60 & $<0.001$ & 0.584 & 1.60 & $<0.001$ & 0.565 \\
\hline \multirow[t]{2}{*}{$d N L R$} & $<2: 1$ & $194(30)$ & 1 & & & 1 & & \\
\hline & $\geqslant 2: 1$ & 445 (70) & 1.61 & $<0.001$ & 0.575 & 1.67 & $<0.001$ & 0.566 \\
\hline
\end{tabular}

Abbreviations: $A \cup C=$ area under receiver-operating characteristic curve; $d N L R=$ derived NLR; HR = hazards ratio; NLR = neutrophil lymphocyte ratio.

The relationship between the NLR, dNLR and Dukes stage in all patients is shown in Figure 2. The relationship between the NLR, dNLR and survival in patients with advanced colorectal cancer (Dukes $C$ and $D$ ) adjusted for age, sex and deprivation is shown in Table 3. These patients were studied, as they are likely to be similar to those entered into chemotherapeutic trials (Chua et al, 2011). On survival analysis $(n=639)$, both the NLR and dNLR were associated with reduced overall and cancer-specific survival independent of age, sex and deprivation (all $P<0.001$ ), with similar hazard ratios and area under the ROC curve (both $P<0.001)$.

\section{DISCUSSION}

The results of the present study show clearly that the NLR and dNLR have similar prognostic value, and that they can be used similarly to predict survival in a large cohort of unselected cancer patients. Furthermore, the NLR and dNLR had similar predictive value, in all cancers as well as advanced colorectal cancer. Recently, Chua et al (2011) reported that the NLR, as a marker of the systemic inflammatory response, predicted clinically meaningful outcomes in patients with advanced colorectal cancer and receiving chemotherapy. Taken together, these results would indicate that the derived NLR is suitable for the examination of risk stratification of patients in chemotherapeutic trials, in particular, colorectal cancer studies.

In the present study, although the ROC analysis of the NLR and dNLR were similar, the prognostic value of different thresholds was examined. It was of interest that in contrast to the most commonly used NLR threshold of 5:1 (Walsh et al, 2005; Gomez et al, 2008; Halazun et al, 2008), in the present study, 4:1 was found to have superior prognostic value in terms of hazard ratio and area under the ROC curve. These results are consistent with the varying NLR threshold reported across and within different tumour types. It is also of interest that the optimal threshold for the dNLR was $2: 1$, an expected shift from that of the NLR explained by the method of derivation. The results of the present study also suggest that the dNLR has considerable potential to be adopted universally as a stratification factor in all current cancer clinical trials. Moreover, if it were shown to have such clinical utility, the dNLR would identify patients who may respond to antiinflammatory interventions.

In the present study, it was of interest that there was a small but persistent superiority of the prognostic value of the NLR over the dNLR. The basis of this observation is not clear. However, in the dNLR, the use of (WBC-neutrophil) in the denominator is broadly mixing two cell types, lymphocytes and monocytes, with possible opposing effects in terms of predictive value. In the normal range, the relative proportion of lymphocytes to monocytes is approximately $6: 1$. In cancer patients, there may be a fall in the absolute proportion of lymphocytes and an increase in the absolute proportion of monocytes. Even so, their relative proportion is unlikely to fall below $3: 1$ even in advanced disease (Leitch et al, 2007). Therefore, WBC - neutrophil is dominated by lymphocytes and is likely to be a reasonable approximation to the lymphocyte fraction, and the potential error introduced by the presence of monocytes in the fraction is therefore likely to be small. Given that different aspects of the differential white cell count have been reported to predict survival (Leitch et al, 2007; Proctor et al, 2011b; Lee et al, 2012), it is possible to derive other ratios, such as the neutrophil: white cell count ratio. However, of the differential white cell count parameters, the neutrophil:lymphocyte ratio has been the most extensively validated, and it was this that we were attempting to recapitulate in the dNLR of the present study. Clearly, where the NLR is available, it should be used. However, there is a wealth of clinical trial data, where only white cell and neutrophil counts have been recorded in computer databases, which could be used to examine, in detail, the clinical value of the haematopoietic tissue-derived systemic inflammatory response. As the present study validates the use of the dNLR, this may help unlock the residual value of such clinical trial databases and encourage the widespread utilisation of a similarly based systemic inflammation-based scores in such settings.

The results of the present study also confirm the hypothesis that a total white cell count may be a useful addition to the currently established prognostic markers of the systemic inflammatory response, such as, C-reactive protein, albumin, neutrophil, and lymphocytes counts and their combinations (Proctor et al, 2011a). It was also of interest that the hazard ratios and areas under the curves were greater from patients sampled after diagnosis compared with patients sampled before diagnosis. These results would suggest that the systemic inflammatory response is a more potent stimulator of cancer progression in established disease. This is consistent with the long-standing observations on the 'seed and soil' nature of cancer progression and metastasis (Fidler and Poste, 2008).

The present cohort has a number of limitations. The patients were selected on the presence of haematological and biochemical variables, and were therefore not necessarily representative of all cancer patients in general. Patients may also have concurrent morbidity, including infection, causing alterations in their haematological variables. It is also recognised that demographic variables, such as race, that appear to influence neutrophil counts were not available. Nevertheless, the optimal prognostic threshold for the NLR in the range of 4-5:1 have been consistently validated in different cancer cohorts (Clarke et al, 2011). It remains to be determined whether a dNLR of $2: 1$ will be similarly validated in different cancer cohorts.

In summary, the results of the present study show that a dNLR, on the basis of a white cell and neutrophil counts, has similar prognostic value to the NLR. Therefore, the universally available dNLR is to be commended for use in the risk stratification of patients undergoing chemotherapy.

\section{ACKNOWLEDGEMENTS}

We acknowledge the academic support and assistance of Drs Talwar, Balmer, O’Reilly and Foulis.

\section{Conflict of interest}

The authors declare no conflict of interest. 


\section{REFERENCES}

Azab B, Bhatt VR, Phookan J, Murukutla S, Kohn N, Terjanian T, Widmann WD (2011) Usefulness of the neutrophil-to-lymphocyte ratio in predicting short- and long-term mortality in breast cancer patients. Ann Surg Oncol 19(1): 217-224

Bishop J, Clark D, Harris V, Stockton D, Sutton M (2004) Deprivation and urban rural measurements in ISD. Summary Report. ISD Geography, Population, Census and Deprivation Group: Edinburgh

Cho H, Hur HW, Kim SW, Kim SH, Kim JH, Kim YT, Lee K (2009) Pretreatment neutrophil to lymphocyte ratio is elevated in epithelial ovarian cancer and predicts survival after treatment. Cancer Immunol Immunother 58: 15-23

Chua W, Charles KA, Baracos VE, Clarke SJ (2011) Neutrophil/lymphocyte ratio predicts chemotherapy outcomes in patients with advanced colorectal cancer. $\mathrm{Br}$ J Cancer 104: 1288-1295

Clarke SJ, Chua W, Moore M, Kao S, Phan V, Tan C, Charles K, McMillan DC (2011) Use of inflammatory markers to guide cancer treatment. Clin Pharmacol Ther 90: 475-478

Colotta F, Allavena P, Sica A, Garlanda C, Mantovani A (2009) Cancerrelated inflammation, the seventh hallmark of cancer: links to genetic instability. Carcinogenesis 30: 1073-1081

Ding PR, An X, Zhang RX, Fang YJ, Li LR, Chen G, Wu XJ, Lu ZH, Lin JZ, Kong LH, Wan DS, Pan ZZ (2010) Elevated preoperative neutrophil to lymphocyte ratio predicts risk of recurrence following curative resection for stage IIA colon cancer. Int J Colorectal Dis 25: 1427-1433

Ferlay J, Shin HR, Bray F, Forman D, Mathers C, Parkin DM. (2010) Cancer Incidence and Mortality Worldwide. IARC CancerBase No. 10 (Internet)International Agency for Research on Cancer: Lyon, France, GLOBOCAN 2008 v1.2.

Fidler IJ, Poste G (2008) The "seed and soil" hypothesis revisited. Lancet Oncol 9: 808

Gomez D, Morris-Stiff G, Toogood GJ, Lodge JP, Prasad KR (2008) Impact of systemic inflammation on outcome following resection for intrahepatic cholangiocarcinoma. J Surg Oncol 97: 513-518

Halazun KJ, Aldoori A, Malik HZ, Al Mukhtar A, Prasad KR, Toogood GJ, Lodge JP (2008) Elevated preoperative neutrophil to lymphocyte ratio predicts survival following hepatic resection for colorectal liver metastases. Eur J Surg Oncol 34: 55-60

Hanahan D, Weinberg RA (2011) Hallmarks of cancer: the next generation. Cell 144: 646-674

Kao SC, Pavlakis N, Harvie R, Vardy JL, Boyer MJ, van Zandwijk N, Clarke SJ (2010) High blood neutrophil-to-lymphocyte ratio is an indicator of poor prognosis in malignant mesothelioma patients undergoing systemic therapy. Clin Cancer Res 16: 5805-5813
Kim HS, Han KH, Chung HH, Kim JW, Park NH, Song YS, Kang SB (2010) Neutrophil to lymphocyte ratio for preoperative diagnosis of uterine sarcomas: a case-matched comparison. Eur J Surg Oncol 36: 691-698

Lee YY, Choi CH, Sung CO, Do IG, Huh S, Song T, Kim MK, Kim HJ, Kim TJ, Lee JW, Kim BG, Bae DS (2012) Prognostic value of pre-treatment circulating monocyte count in patients with cervical cancer: comparison with SCC-Ag level. Gynecol Oncol 124: 92-97

Leitch EF, Chakrabarti M, Crozier JE, McKee RF, Anderson JH, Horgan PG, McMillan DC (2007) Comparison of the prognostic value of selected markers of the systemic inflammatory response in patients with colorectal cancer. Br J Cancer 97: 1266-1270

Ohno Y, Nakashima J, Ohori M, Hatano T, Tachibana M (2010) Pretreatment neutrophil-to-lymphocyte ratio as an independent predictor of recurrence in patients with nonmetastatic renal cell carcinoma. J Urol 184: 873-878

Proctor MJ, Morrison DS, Talwar D, Balmer SM, Fletcher CD, O’Reilly DS, Foulis AK, Horgan PG, McMillan DC (2011a) A comparison of inflammation-based prognostic scores in patients with cancer. A Glasgow Inflammation Outcome Study. Eur J Cancer 47: 2633-2641

Proctor MJ, Morrison DS, Talwar D, Balmer SM, O'Reilly DS, Foulis AK, Horgan PG, McMillan DC (2011b) An inflammation-based prognostic score (mGPS) predicts cancer survival independent of tumour site: a Glasgow Inflammation Outcome Study. Br J Cancer 104: 726-734

Proctor MJ, Talwar D, Balmar SM, O'Reilly DS, Foulis AK, Horgan PG, Morrison DS, McMillan DC (2010) The relationship between the presence and site of cancer, an inflammation-based prognostic score and biochemical parameters. Initial results of the Glasgow Inflammation Outcome Study. Br J Cancer 103: 870-876

Sarraf KM, Belcher E, Raevsky E, Nicholson AG, Goldstraw P, Lim E (2009) Neutrophil/lymphocyte ratio and its association with survival after complete resection in non-small cell lung cancer. J Thorac Cardiovasc Surg 137: 425-428

Sharaiha RZ, Halazun KJ, Mirza F, Port JL, Lee PC, Neugut AI, Altorki NK, Abrams JA (2011) Elevated preoperative neutrophil:lymphocyte ratio as a predictor of postoperative disease recurrence in esophageal cancer. Ann Surg Oncol 18: 3362-3369

Shimada H, Takiguchi N, Kainuma O, Soda H, Ikeda A, Cho A, Miyazaki A, Gunji H, Yamamoto H, Nagata M (2010) High preoperative neutrophillymphocyte ratio predicts poor survival in patients with gastric cancer. Gastric Cancer 13: 170-176

Walsh SR, Cook EJ, Goulder F, Justin TA, Keeling NJ (2005) Neutrophillymphocyte ratio as a prognostic factor in colorectal cancer. J Surg Oncol 91: 181-184

This work is published under the standard license to publish agreement. After 12 months the work will become freely available and the license terms will switch to a Creative Commons Attribution-NonCommercial-Share Alike 3.0 Unported License. 